Classification

Physics Abstracts

$46.30 \mathrm{P}-04.75 \mathrm{D}$

\title{
Drag on a Tethered Chain Moving in a Polymer Melt
}

\author{
A. Ajdari $\left({ }^{1}\right)$, F. Brochard-Wyart $\left({ }^{2}\right)$, C. Gay $\left({ }^{3}\right)$, P.G. de Gennes $\left({ }^{3}\right)$ and J.L. Viovy $\left({ }^{1}\right)$ \\ ( ${ }^{1}$ ) ESPCI, Physico-Chimie Théorique, 10 rue Vauquelin, 75231 Paris Cedex 05, France \\ $\left({ }^{2}\right)$ PSI, Institut P. et M. Curie, 11 rue P. et M. Curie, 75231 Paris Cedex 05, France \\ $\left({ }^{3}\right)$ Collège de France, 1 place M. Berthelot, 75231 Paris Cedex 05, France \\ (Received 17 January 1995, accepted 25 January 1995)
}

\begin{abstract}
A chain of $N$ monomers is attached to a small colloïdal particle, and is pulled (at a velocity $V$ ) inside a polymer melt (chemically identical, with $P$ monomers per chain). The main parameter for this problem is the number $X(V)$ of $P$ chains entangled with the $(N)$ chain. Earlier estimates of $X$ are criticized, and a new form is proposed: at large $N\left(N>N_{\mathrm{e}}^{2}\right)$, we are led to a "Stokes" regime, $X=N^{1 / 2}$, while at smaller $N\left(N<N_{e}^{2}\right)$, we find a "Rouse" regime, $X=N / N_{\mathrm{e}}$ (where $N_{\mathrm{e}}$ is the number of monomers per entanglement).
\end{abstract}

\section{Introduction}

The motion of a long, tethered chain ( $N$ monomers) inside a polymer melt $(P)$ is special: the $N$ chain cannot reptate inside the $(P)$ matrix [1]. This occurs in star polymers, and also in two recent experimental situations (Fig. 1):

a) The $N$ chain is grafted to a colloïdal particle (of size smaller than the coil radius $R_{N}$ of the $N$ chain). The particle can be driven by sedimentation or by optical tweezers (Fig. 1a).

b) The $N$ chain is grafted on a flat wall, and the $(P)$ melt flows tangentially to the wall (Fig. 1b). (In the following, we assume that the grafting density is very small: no coupling between different $N$ chains).

Problem b) was first considered theoretically (for the low $V$ limit) in reference [2]. The starting point is that a certain number $X(V)$ of $P$ chains are entangled with the $N$ chain. The resulting friction is estimated as follows [3]:

Assume that the $N$ chain has moved by a distance $D^{*}$ equal to the diameter of an Edwards tube [4] $D^{*}=N_{\mathrm{e}}^{1 / 2} a$, where $N_{\mathrm{e}}$ is the number of monomers per entanglement, and $a$ the monomer size. (We take $N_{\mathrm{e}}<N \leq P$ ). To allow for this motion of the $N$ chain, each $(P)$ chain entangled with $(N)$ must move, along its own tube (of length $L_{\mathrm{t}}$ ) by something like $L_{t}$. 


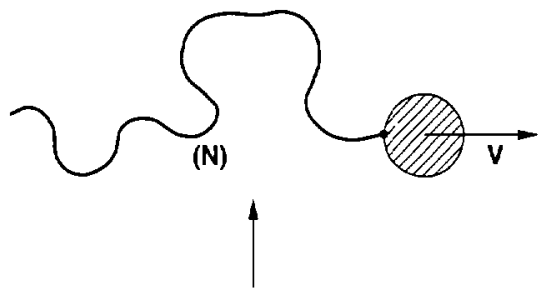

(P) matrix

(a)

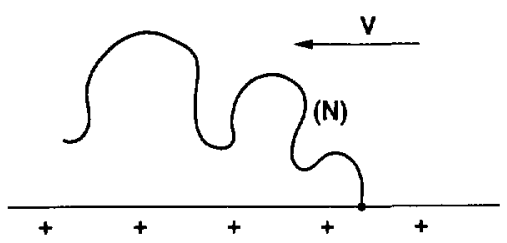

(b)

Fig 1. - Two examples of a tethered chain $(N)$ pulled inside a polymer melt $(P)$, a) Sedımentation (at optical tweezer action) on a colloid grain. b) Shear flow near a weakly grafted surface.

Thus the sliding velocity $V_{\mathbf{s}}$ of this $P$ chain is not the translational velocity $V$, but is much larger:

$$
V_{\mathrm{s}} \cong V \frac{L_{\mathrm{t}}}{D^{*}}=V \frac{P}{N_{\mathrm{e}}}
$$

The dissipation $T \dot{S}$ due to the motion of the tethered chain corresponds to $X(P)$ chains moving at velocity $V_{\mathrm{s}}$ in the ambient melt.

$$
T \dot{S}=X \zeta_{1} P V_{\mathrm{s}}^{2}=f V
$$

where $\zeta_{1} P$ is the tube friction coefficient of one $(P)$ chain, and $f$ the drag force. Comparing the two expressions of $T \dot{S}$ we get:

$$
f=V X(V) a \eta_{p}
$$

where $\eta_{p}=\zeta_{1} a^{-1} P^{3} N_{\mathrm{e}}^{-2}$ is the reptation viscosity of the $(P)$ melt.

The crucial question is thus to find $X(V)$. Even in the simplest $(V \rightarrow 0)$ limit, where the $(N)$ chain is an unperturbed coil, this problem is difficult, and different answers have been proposed at different times [2,3]. In Section 2, we reanalyse the problem, using what we call the binary entanglement model. In Section 3, we compare this with a "collective" entanglement model. Section 4 extends our ideas to higher velocities

\section{Binary Entanglements}

At low velocities, the $N$ chain is an unperturbed coil, of size $R_{N} \cong N^{1 / 2} a$ and volume $R_{N}^{3}$. It experiences in average an entanglement every $N_{\mathrm{e}}$ monomers. Each $(P)$ chain intersecting this 


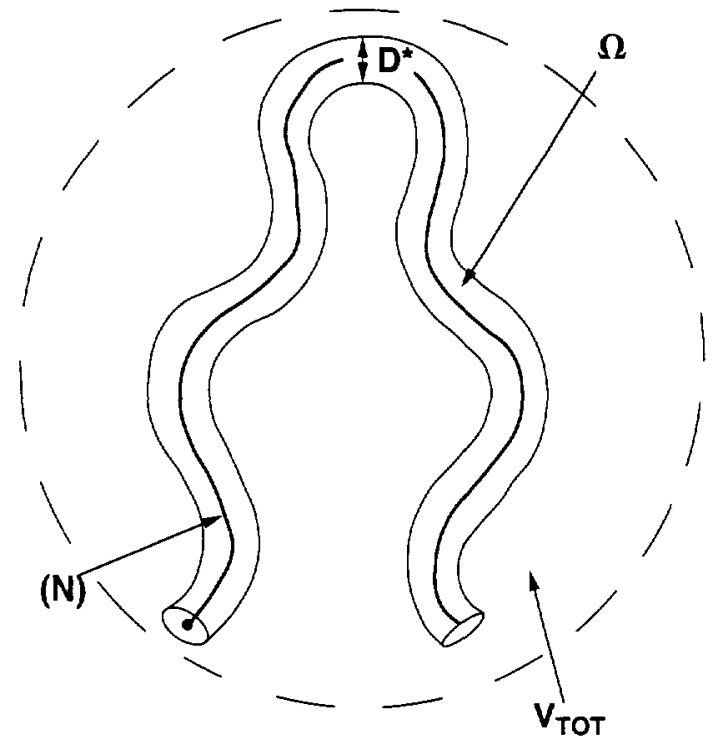

Fig. 2 - The tethered chan at low velocities. coil volume $V_{\text {tot }}$ and Edwards tube of volume $\Omega$.

volume uses $\sim N$ monomers in this region. Thus the number of $(P)$ chains which overlap with the $(N)$ coil is $R_{N}^{3} / N a^{3}=N^{1 / 2}$.

In reference [2] we simply assumed that all the $P$ chains are entangled with the $(N)$ chain, i.e. $X(V \rightarrow 0)=N^{1 / 2}$.

The Edwards tube surrounding the $N$ chain (Fig. 2) is a sequence of $N / N_{\mathrm{e}}$ blobs with diameter $D^{*}$ and total volume.

$$
\Omega=N / N_{\mathrm{e}}\left(D^{*}\right)^{3}
$$

One of the $P$ chains intersecting the volume $R_{N}^{3}$ has $N \Omega / R_{N}^{3}$ monomers inside the tube. The number of blobs visited by the $P$ chain is thus:

$$
b=\left(N / N_{\mathrm{e}}\right)\left(\Omega / R_{N}^{3}\right)=\left(N / N_{\mathrm{e}}\right)^{1 / 2}
$$

Inside one blob, $N_{\mathrm{e}}^{1 / 2}$ chains coexist (including the $N$ chain). In our binary entanglement model, we assume that a constraint is associated with a pair of chains inside the blob. The total number of pairs is $1 / 2\left(N_{\mathrm{e}}^{1 / 2}\right)^{2} \sim N_{\mathrm{e}}$. Thus, the probability that any given pair of chains inside the volume do entangle, is only $N_{\mathrm{e}}^{-1 / 2}$. The number $c$ of constraints between one $(P)$ chain and the $(N)$ chain is then:

$$
c=b N_{\mathrm{e}}^{-1 / 2}=N^{1 / 2} / N_{\mathrm{e}}
$$

We are thus led to distinguish two very different regimes:

1) $N>N_{\mathrm{e}}^{2}$. In this case $c$ is larger than unity: all the $N^{1 / 2}(P)$ chains which intersect the coil do entangle with $(N)$. Thus the simple guess of reference [2] is confirmed:

$$
X=N^{1 / 2}
$$

We call this the Stokes regime, because the friction force (Eq. (3)) has the scaling form corresponding to a Stokes sphere (radius $N^{1 / 2} a$ ) inside a liquid of viscosity $\eta_{p}$. 
2) $N<N_{\mathrm{e}}^{2}$. In this case $c$ is smaller than unity, and we cannot use equation (5). When $c \ll 1$, we may say that the probability of entanglement between one $(P)$ chain (intersecting the coil) and the $(N)$ chain is $c$. Thus:

$$
X=N^{1 / 2} c=N / N_{\mathrm{e}}
$$

The friction experienced by the tethered chain is then linear in $N$. Although we deal with an entangled system, the $(N)$ chain is thus described by a Rouse model [6], but the Rouse friction coefficient is proportional to the melt viscosity (as can be seen from Eqs. (3)-(8)).

\section{The Collective Entanglement Model}

We now wish to describe an opposite limit, where one entanglement site (a blob of diameter $D^{*}$ ) is pictured as a very complex knot, involving $N_{\mathrm{e}}^{1 / 2}$ chains; the knot is such that eliminating one chain from it removes the constraint. Then the number of constraints released if one $P$ chain moves out of the volume $R_{N}^{3}$ is $b$, and is larger than unity. All the $N^{1 / 2}$ " $P$ - chains" are thus coupled to the $N$ chain.

In this model, it would be enough to select a subset of $\left(N / N_{\mathrm{e}}\right)^{1 / 2}$ " $P$-chains" and move them out, to relax the $N$ chain: since $\left(N / N_{\mathrm{e}}\right)^{1 / 2} b=N / N_{\mathrm{e}}$ is the total number of constraints to be removed. This remark leads to the prediction of reference [5]. However, we do not think that this approach is realistic. The $N$ chain, when it moves, has no way of selecting a subset of releasing chains: it drags all of them.

Thus we are led to say that in the collective entanglement model, $X=N^{1 / 2}$, and the Stokes model holds for all values of $N$.

\section{High Velocities in the Binary Entanglement Model}

Under strong flows, and in the simplest picture [7], the $N$ chains become elongated into a cigar shape, with diameter $D$ and length $L=R_{N}^{2} / D$. A more sophisticated description has been constructed [5] but is essentially equivalent in practice. We have to distinguish two regimes:

4.1. Partial striction. $-R_{N}>D>D^{*}$. Here, a simple repetition of our discussion in Section 2 gives:

$$
c=D /\left(a N_{\mathrm{e}}\right)
$$

If $N<N_{\mathrm{e}}^{2}$, we always stay in the Rouse regime $\left(X=N / N_{\mathrm{e}}\right)$.

If $N>N_{\mathrm{e}}^{2}$, we find a crossover from strong coupling to Rouse upon increasing the velocity (decreasing $D$ ).

4.2. Marginal REgime. - Here the cigar diameter $D$ becomes comparable to $D^{*}$, and the number of entanglements realised by the $N$ chain can become smaller than $N / N_{\mathrm{e}}$. The marginal value of $X\left(X=X^{*}\right)$ is in fact fixed by the force balance: the stretching force required to reach $D^{*}$ is [8]:

$$
\frac{k T}{D^{*}}=X^{*}(V) a \eta_{p} V
$$

and thus $X^{*}$ is inversely proportional to the velocity. It may be checked that for $V=V^{*}$ (the onset velocity for the marginal regime) $X^{*}=N / N_{\mathrm{e}}$ as expected in the Rouse regime and:

$$
V^{*}=k T N_{\mathrm{e}}^{1 / 2} /\left(N \eta_{p} a^{2}\right)
$$




\section{Concluding Remarks}

1) The results of the binary entanglement model can be summarized as follows (for the low velocity limit): the number of entangled chains is either $N^{1 / 2}$ (the number of ambient chains intersecting the mushroom) or $N / N_{\mathrm{e}}$ (the number of constraints acting on the $N$ chain). Each of them is an upper bound for $X$, and thus $X$ is the smaller of the two.

2) In the collective entanglement model, we are led to $X=N^{1 / 2}$. (See also [9]). But we do not think that the collective model is fully realistic: complex knots may play a role, but may not dominate the behaviour.

The real situation is probably intermediate between the binary and the collective model, as suggested by the concentration dependence of the modulus of a melt plastified with moderate amount of solvent. The binary model predicts $G_{0} \sim c^{2}$; the collective entanglement approach predicts $G_{0} \sim c^{3}$; and the typical experimental exponent is $G_{0} \sim c^{2.2 \sim 24}$ (see, e.g., Ref. [10] and Refs. therein). Thus, for many applications, one can stick to the binary model, which is conceptually simpler.

3) It is instructive to discuss the whole distribution function $p(n)$ for the number of entanglements between one given $P$ chain (intersecting the mushroom) and the $N$ chain. Using mean-field arguments, one obtains a Poisson distribution:

$$
p(n)=\exp \left(-N^{1 / 2} / N_{\mathrm{e}}\right)\left(\frac{N^{1 / 2}}{N_{\mathrm{e}}}\right)^{n} \frac{1}{n !}
$$

This gives:

$$
\begin{aligned}
X & =N^{1 / 2}[1-p(0)] \\
& =N^{1 / 2}\left[1-\exp \left(-N^{1 / 2} / N_{\mathrm{e}}\right)\right]
\end{aligned}
$$

Equation (12) is a useful interpolation between equations (7) and (8).

\section{Acknowledgments}

We have greatly benefited from discussions with J.F. Joanny and E. Raphaël.

\section{References}

[1] de Gennes P.G., J. Phys. France 36 (1975) 1199; Doi M. and Kuzuu N., J. Polym. Scz. Polym. Lett. 18 (1980) 775; Pearson D and Helfand E., Macromolec. 17 (1984) 888.

[2] Brochard-Wyart F., de Gennes P.G. and Pincus P., C.R. Acad. Scr. Paris 314 II (1992) 873.

[3] de Gennes P.G., Mater. Res. Soc. Bull. (1991) 20.

[4] For a review, see: Edwards S.F., Molecular Fluids, R. Balıan and G. Weill, Eds. (Gordon and Breach, NY, 1976).

[5] Ajdari A., Brochard F., de Gennes P.G., Leibler L., Viovy J L. and Rubinsteın M., Physica A204 (1994) 17. See in particular equation (7) of this reference.

[6] For a recent presentation of Rouse friction see: de Gennes P.G., Introduction to Polymer Dynamics (Academia del Lincel Ed., Rome, 1990).

[7] Brochard F. and de Gennes P.G, Langmuır 8 (1992) 3033.

[8] Pincus P., Macromolec. 9 (1976) 386.

[9] Brochard F., Ajdari A., Leibler L, Rubinstein M. and Viovy J.L., Macromolec. 27 (1994) 803.

[10] Colby R.H., Rubinstein M. and Viovy J.L., Macromolec. 25 (1992) 996. 